\title{
Cartographie quantitative de l'érosion des sols par approche SIG/RUSLE dans la Commune de Karangasso vigué (Burkina Faso)
}

\author{
Blaise OUEDRAOGO ${ }^{1 *}$, Oumar KABORE ${ }^{1}$ et Martine KABORE ${ }^{2}$ \\ ${ }^{1}$ Institut de l'Environnement et de Recherches Agricoles, CREAF de Kamboinsé Ouagadougou, 01 BP 476 \\ Ouaga 01, Burkina Faso. \\ ${ }^{2}$ Université Joseph Ki-Zerbo, Ouagadougou, Burkina Faso. \\ *Auteur correspondant; E-mail : blaise32fr@yahoo.fr
}

\section{RESUME}

La dégradation des conditions climatiques a provoqué un afflux massif de populations du nord vers l'ouest du Burkina Faso, créant de nouveaux fronts pionniers agricoles. De cette croissance démographique, s'ajoutent des systèmes de productions agricoles inadaptés qui ont donné lieu à une dégradation de la végétation et augmenter l'érosion hydrique dans cette partie du pays. L'objectif de cette étude est de cartographier les principaux paramètres explicatifs de l'érosion hydrique afin d'établir une carte de risque d'érosion dans la commune de Karangasso Vigué. La méthodologie s'appuie sur une modélisation basée sur l'intégration des principaux facteurs de l'érosion hydrique dans un système d'information géographique. Les résultats obtenus mettent en relief l'impact érosif de chaque facteur et établissent une hiérarchisation des zones à risque d'érosion hydrique du site d'étude. La carte résultante montre des valeurs de perte de terre de 0 à 1,57 t/ha/an sur une superficie de 187196,54 ha et de 8,42 à 36,35 t/ha/an couvrant une superficie de 362,11 ha. Cette carte est un outil d'aide à la décision pour les autorités au niveau local et pour les projets d'aménagement agro-sylvo-pastoraux afin d'orienter les différentes actions d'intervention de conservation des eaux et des sols. (C) 2019 International Formulae Group. All rights reserved

Mots clés: Erosion hydrique, SIG, RUSLE. Karangasso Vigué.

\section{Quantitative mapping of soil erosion using a GIS/RUSLE approach in the district of Karangasso Vigué (Burkina Faso)}

\begin{abstract}
The deterioration of climatic conditions has caused a massive influx of people from the north to the west of Burkina Faso, creating new agricultural pioneer fronts. This population growth is compounded by inadequate agricultural production systems that have led to vegetation degradation and increased water erosion in this part of the country. The objective of this study is to map the main explanatory parameters of water erosion in order to establish an erosion risk map in the commune of Karangasso Vigué. The methodology is based on modelling based on the integration of the main factors of water erosion into a geographic information system. The results obtained highlight the erosive impact of each factor and establish a hierarchy of the water erosion risk areas of the study site. The resulting map shows land loss values from 0 to $1.57 \mathrm{t} / \mathrm{ha} / \mathrm{year}$ over an area of 187196.54 ha and from 8.42 to $36.35 \mathrm{t} / \mathrm{ha} /$ year over an area of 362.11 ha. This map is a decisionmaking tool for local authorities and agro-sylvo-pastoral development projects to guide the various water and soil conservation intervention actions.
\end{abstract}

(C) 2019 International Formulae Group. All rights reserved

Keywords: Water erosion, GIS, RUSLE. Karangasso Vigué. 


\section{INTRODUCTION}

Le Sahel est une zone extrêmement sensible aux variations climatiques (Gasse, 2006; Ozer et al., 2010). Cette variabilité climatique entraine de profondes modifications des paysages, avec la réduction, voire la disparition du couvert végétal (Hountondji, 2008).

L'Ouest du Burkina Faso connait en effet, depuis des dizaines d'années des mutations spatiales majeures, qui ont des répercussions importantes sur les deux principales activités rurales régionales que sont l'agriculture et l'élevage (Kohio et al., 2017 ; Gonin et Tallet, 2012). Cette région a connu aussi des rythmes de croissance démographique très élevés (10\% par an) suite à la conjonction de l'accroissement naturel et de l'arrivée de nouveaux migrants (Nana, 2018). Cette croissance de la population a entrainé une augmentation des espaces cultivables avec une forte diminution de la pratique de la jachère (Fournier $\mathrm{J}$ et al) et d'une fragmentation de l'espace suite à l'ouverture de nombreux nouveaux champs (Ouédraogo et al., 2015). Malgré les conditions pluviométriques favorables dont bénéficie la zone ouest du pays, on assiste à une baisse continue de la fertilité des sols et plus particulièrement de leur teneur en matière organique compromettant ainsi la durabilité des systèmes de productions agro-sylvopastorales. (Da et al., 2008; Ouandaogo et al., 2016). L'érosion hydrique constitue l'un des principaux facteurs de dégradation des terres et des problèmes de l'environnement particulièrement au sahel (Hamimed, 2015 ; Avakoudjo et al., 2015). Elle est causée généralement par la croissance démographique et par les effets des changements climatiques. L'agressivité des pluies et surtout les mauvaises pratiques culturales dégradent le sol et entrainent des pertes de terre (Mokhtari, 2017).

C'est le constat actuel dans la commune de Karangasso Vigué où les sols sont soumis à une forte pression anthropique et deviennent très sensibles à la dégradation avec une baisse de leur fertilité.
L'objectif de cette étude est de cartographier les principaux paramètres explicatifs de l'érosion hydrique et d'établir une carte de vulnérabilité de l'érosion dans la commune de Karangasso-Vigué.

\section{MATERIEL ET METHODES Le site d'étude}

La commune rurale de KarangassoVigué est située dans la province du Houet localisée dans la région des Hauts Bassins. D'une superficie de $2026 \mathrm{~km}^{2}$, elle est située entre $3^{\circ} 36^{\prime}$ et $4^{\circ} 10^{\prime}$ de longitude Ouest et entre $10^{\circ} 42^{\prime}$ et $11^{\circ} 10^{\prime}$ de latitude Nord. Elle s'étend sur $61 \mathrm{~km}$ environ d'Est à l'Ouest et sur $53 \mathrm{~km}$ environ du Nord au Sud.

Elle est limitée :

- au Nord par les communes rurales de Koumbia (Province du Tuy) et de Lena (Houet),

- au Sud, par les communes rurales de Sidéradougou et de Ouo (provinces de la Comoé),

- à l'Est par le fleuve Bougouriba et la commune rurale de Bondigui (province de la Bougouriba),

- à l'Ouest, par la commune rurale de Péni et la commune urbaine de Bobo-Dioulasso (province du Houet).

Karangasso-Vigué, chef-lieu de la commune, est à $57 \mathrm{~km}$ du chef-lieu de la province du Houet (Bobo-Dioulasso), auquel l'on accède par la route nationale $\mathrm{RN}^{\circ} 1$.

Karangasso-Vigué est à $9 \mathrm{~km}$ de la RN27 qui relie Yéguérésso (Bobo-Dioulasso) à Diébougou à partir du village de Klésso. La localisation de la commune rurale de Karangasso-Vigué est présentée par la Carte 1.

Karangasso Vigué est un front pionnier agricole qui connait depuis quelques décennies une forte occupation humaine pour l'agriculture et l'élevage. C'est un territoire représentatif de la modification du paysage par les activités humaines qui entrainent des bouleversements dans la structure de la végétation et des sols.

La commune de Karangasso Vigué appartient au climat de type sud-soudanien délimitée par les isohyètes 1000 et $1200 \mathrm{~mm}$. 
Les pluies sont distribuées de façon unimodale avec un maximum généralement observé en août. Les précipitations se concentrent au milieu de la saison humide et sur moins de jours pluvieux par rapport aux dernières décennies. A la réduction des totaux annuels s'ajoute un régime de précipitations plus violentes, avec des allures agressives. Ces caractéristiques climatiques jouent alors un rôle très important dans l'érosion hydrique.

\section{Les méthodes et approches de la recherche}

L'équation de Wischmeier révisée est combinée avec les techniques de SIG pour analyser le taux de perte brute de sol et pour évaluer la distribution spatiale des taux de perte de sol sur les différentes occupations des sols (Yjjou et al., 2014). L'érosion hydrique est un phénomène complexe qui résulte de trois phases: détachement, transport et dépôt causés par l'action en général combinée de la pluie et du ruissellement, et dont l'expression varie en fonction de la résistance du milieu (sol, couvert végétal, techniques culturales) et de la topographie. Quelle que soit l'échelle d'étude, du mètre carré au bassin versant de centaines de $\mathrm{km}^{2}$, on retrouve partout ces trois phases de l'érosion, mais avec des intensités différentes; d'où la diversité des facteurs de l'érosion en fonction des phases dominantes que sont le détachement, le transport et le dépôt (Simonneaux et al., 2015).

Pour atteindre les objectifs de l'étude, la démarche méthodologique choisie repose sur l'utilisation des données de la télédétection qui permet une connaissance spatialisée des facteurs de l'érosion (érosivité des précipitations, occupation du sol, importance du couvert végétal...); et sur l'usage du système d'information géographique (SIG) par des opérations d'analyse et de modélisation des processus d'érosion des terres dans la zone de recherche

\section{La technique et les outils de collecte des données}

La technique de collecte des données comporte deux phases: la recherche documentaire et les enquêtes terrains.

\section{Les enquêtes de terrain}

Les enquêtes de terrain ont permis d'administrer un questionnaire à 150 ménages des six (06) villages de la commune rurale de Karangasso-Vigué (Tableau 1). Des guides d'entretien ont aussi été adressés aux responsables de la mairie et aux services techniques déconcentrés de l'Etat (environnement, agriculture et élevage). Ils portaient sur la dynamique des ressources naturelles (eau, sol, végétation) du sous bassin versant, et les stratégies de gestion et de conservation mises en œuvre. Et enfin, des observations directes sur le terrain ont été faites et ont permis de faire l'état de l'érosion des sols, de faire des prises de vues photographiques et choisir des sites d'entrainement pour le traitement des images satellites.

\section{Les techniques de traitement et d'analyse des données}

Le traitement a consisté au dépouillement des questionnaires et à leur saisie. Les données des enquêtes ont été saisies sur le logiciel statistique Sphinx. Les images satellites ont été traitées avec le logiciel de traitement d'image Envi 4.7. L'acquisition des données primaires par le biais de l'imagerie satellite ont fait l'objet d'intégration dans les logiciels Arc GIS 10.3 et QGIS 2.8. La réalisation des cartes factorielles de perte en sol et de carte de synthèse a suivi les étapes suivantes:

- Extraction de la couche raster SRTM de la zone de recherche;

- génération des courbes de niveau d'équidistance 10 mètres en utilisant la commande «isoline» de spatial analyst Tools, avec comme donnée en entrée «la couche Raster »;

- l'outil «topo to raster » pour générer le Modèle Numérique de Terrain (MNT);

- détermination de l'inclinaison et de la direction de la pente à l'aide du MNT;

- prétraitement de l'image satellite: composition colorée, choix des sites d'entrainement, classification, vérification de la séparabilité et de l'indice Kappa ; 
- Post-classification : application d'un filtre médian, lissage des classes, homogénéisation et vectorisation;

- l'analyse des sols et la carte pédologique ont permis le calcul du facteur d'érodibilité du sol et l'élaboration de la carte du facteur $\mathrm{K}$;

- L'ortho photo et la visite terrain ont permis de déterminer les pratiques antiérosives mises en œuvre par la population ;

- le croisement des cartes des principaux facteurs intervenant dans l'érosion hydrique des sols a donné la carte potentielle en pertes de terre en tout point de la zone de recherche.

L'équation universelle de pertes en sol (Wischmeier et Smith, 1978) s'applique à la quantification des pertes en sol par érosion en nappe. C'est une fonction multiplicative des cinq facteurs qui contrôlent l'érosion hydrique que sont : agressivité climatique, érodibilité des sols, inclinaison et longueur de pente, occupation des sols et pratique antiérosive. $\mathrm{La}$ formule est la suivante $A=R$. K. LS. C. $P$ où A est le taux annuel de perte en sol en t/ha/an, $\mathrm{R}$ est le facteur de l'érosivité des pluies; il correspond à la moyenne annuelle des sommes des produits de l'énergie cinétique de la pluie par son intensité en $30 \mathrm{mn}$ consécutives; il est exprimé en MJ.mm / ha. h. an. Quant à l'érodibilité $\mathrm{K}$ des sols, il dépend de la granularité, de la quantité de matière organique, de la perméabilité et de la structure du sol; il est exprimé en tonne par hectare et par heure t. ha. h / ha. MJ.mm. LS est un facteur sans dimension qui représente l'inclinaison ( $\mathrm{S}$ en \%) et la longueur de pente ( $\mathrm{L}$ en $\mathrm{m})$. Enfin, $\mathrm{C}$ et $\mathrm{P}$ sont des facteurs adimensionnels qui représentent respectivement l'effet de la couverture végétale, puis un rapport qui tient compte des techniques culturales antiérosives telles que le labour en courbes de niveau. La commande « raster calculator » de spatial analyst tools du logiciel Arc Gis 10.3 a été utilisée pour cette opération. Un résumé des traitements intermédiaires qui interviendront dans la réalisation des cartes factorielles des pertes en sol ainsi que de la carte synthèse est faite à travers l'organigramme méthodologique de l'intégration du modèle USLE dans un SIG (Figure 1).

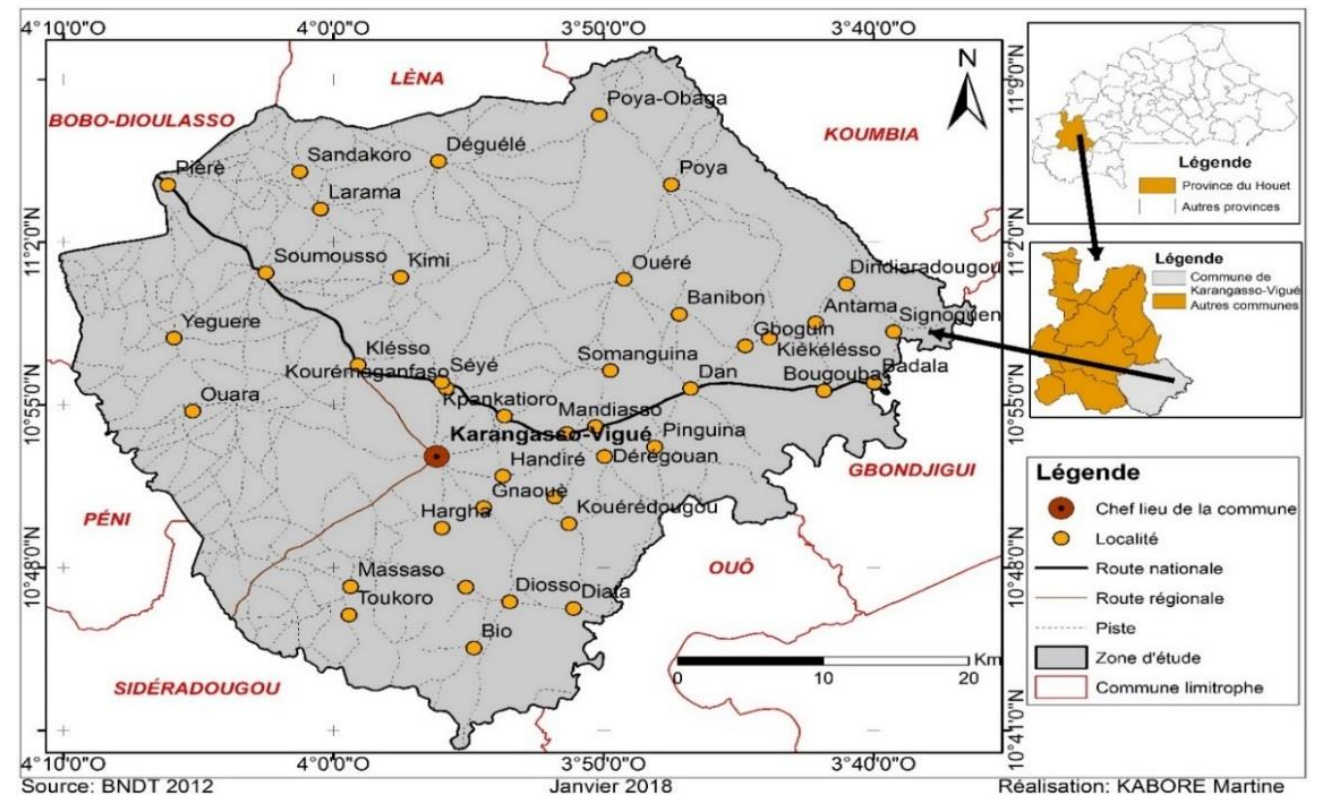

Carte 1 : Localisation de la zone d'étude. 
Tableau 1 : Répartition de l'échantillonnage spatial.

\begin{tabular}{|c|c|c|c|c|}
\hline Numéro & Localités & $\begin{array}{l}\text { Nombre } \\
\text { d'habitants }\end{array}$ & $\begin{array}{l}\text { Nombre de } \\
\text { ménages }\end{array}$ & Ménages enquêtés \\
\hline 1 & Karangasso-Vigué & 5660 & 831 & 25 \\
\hline 2 & Dan & 7169 & 1209 & 25 \\
\hline 3 & Poya & 5456 & 827 & 25 \\
\hline 4 & Dèguèlin & 5813 & 836 & 25 \\
\hline 5 & Soumousso & 6148 & 1013 & 25 \\
\hline 6 & Yéguéré & 3433 & 533 & 25 \\
\hline
\end{tabular}

Source : enquête de terrain

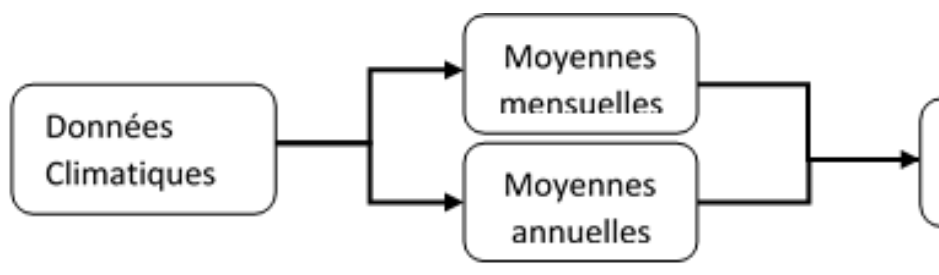

Facteurs d'érosivité de

la pluie $R$
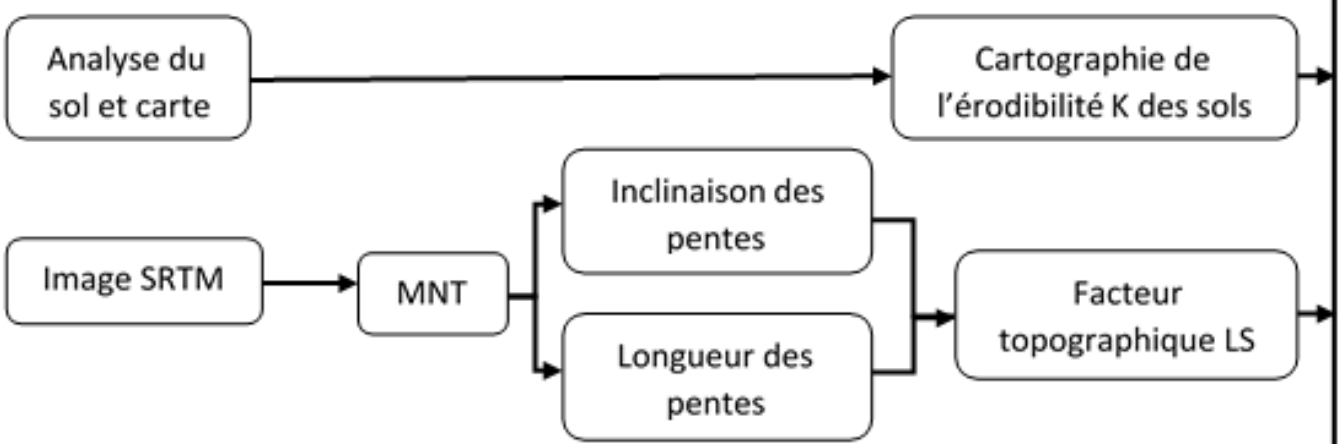

\section{Occupation du sol}

Facteur du couvert végétal C

\section{Photographie aérienne \\ + Visite terrain}

\section{Pratiques antiérosives (P)}

\section{Carte des pertes en sol en (t/ha/an) : A=R*K*LS*C*P}

Figure 1 : Organigramme méthodologique du modèle RUSLE appliqué. 


\section{RESULTATS}

Les sols opposent une résistance à l'érosion hydrique surtout au regard de l'agressivité climatique, du couvert végétal et des pratiques agricoles.

L'utilisation du logiciel ArcGis a permis de calculer et de quantifier l'importance de chaque facteur dans le processus de l'érosion hydrique. Une spatialisation de ces différents facteurs a été réalisée et a permis d'obtenir les cartes factorielles de l'érosion hydrique du site de l'étude. Le croisement de l'ensemble de ces facteurs a donné lieu à la carte synthèse sur les pertes en terres.

\section{Le facteur de la pente topographique (LS).}

Le facteur topographique représente l'inclinaison ( $\mathrm{S}$ en \%) et la longueur de pente $(\mathrm{L}$ en $\mathrm{m})$; En utilisant cette formule mathématique de Wischmeier, le facteur topographique (LS) a été calculé à l'échelle du site d'étude à partir de la carte de l'inclinaison des pentes et de leur longueur obtenues à partir de traitements sous SIG du MNT de la zone d'étude. La Carte 2 représente le facteur topographique LS de la zone de recherche.

Une reclassification en cinq classes a été réalisée, allant des classes de très faible pente occupant une superficie de 179287,60 hectares correspondant à $92,49 \%$ de la superficie totale, aux classes de pente très élevée s'étalant sur une superficie de 126,75 hectares soit $0,07 \%$ de la superficie totale de la zone de recherche (Tableau 2). Le facteur de l'érodibilité $\mathrm{K}$ des sols.

La Carte 3 représente le facteur d'érodibilité $\mathrm{K}$ des sols de la commune de Karangasso-Vigué. La résolution spatiale de cette image matricielle est de $30 \mathrm{~m}$. Cinq classes du facteur $\mathrm{K}$ ont été réalisées pour exprimer l'étendue de la variabilité de l'érodibilité. La classification a été réalisée suivant la méthode des seuils naturels (méthode de jenks). Par conséquent, les valeurs illustrées sur cette carte doivent être considérées comme des valeurs relatives à l'échelle de la commune.

$\mathrm{La}$ valeur moyenne du facteur d'érodibilité $\mathrm{K}$ atteint $0,52 \mathrm{t} \mathrm{h}$ ha $\mathrm{MJ}^{-1} \mathrm{ha}^{-1}$ $\mathrm{mm}^{-1}$ sur l'ensemble de la zone de recherche. Les zones accusant une très faible érodibilité $\left(\mathrm{K}<0,15 \mathrm{t} \mathrm{h}\right.$ ha $\mathrm{MJ}^{-1} \mathrm{ha}^{-1} \mathrm{~mm}^{-1}$ ) sont représentées par les lithosols sur cuirasse et les lithosols sur roche. Ces régions sont caractérisées par une lithologie imperméable regroupant les faciès Karstiques et sableux. Cette classe occupe une superficie de 16 708,77 hectares soit $8.57 \%$ de la superficie totale de la zone de recherche. La zone à érodibilité très élevée $\left(0,61\right.$ à $0,75 \mathrm{t} / \mathrm{h} / \mathrm{ha} / \mathrm{MJ}^{-1}$ $\mathrm{ha}^{-1} \mathrm{~mm}^{-1}$ ) est représentée par les sols ferrugineux tropicaux lessivés indurés et ceux ferralitiques. Cette classe est la plus représentative de la zone et occupe une superficie de 118 231,94 hectares soit 60,64\% de la superficie totale (Tableau 3).

En général, les sols qui affichent une plus grande résistance à l'érosion sont ceux dans lesquels l'eau s'infiltre plus rapidement, ceux qui sont riches en matière organique et ceux dont la structure est améliorée. Les sables ont tendance à être moins vulnérables à l'érosion que les limons, les sables très fins et certains sols argileux.

\section{Le facteur couvert végétal (facteur C)}

Le couvert végétal protège le sol de deux manières en améliorant l'infiltration et les propriétés physiques, chimiques du sol et maintenant la cohésion des matériaux. D'autre part, il brise l'énergie cinétique des gouttes de pluie et intercepte une partie des précipitations.

Le processus d'érosion est étroitement, lié au mode d'occupation du sol, qui contribue largement à son aggravation ou à son atténuation. L'occupation du sol détermine le degré de protection des sols.

La carte d'occupation des terres (Carte 4) résultante du traitement de l'image Landsat TM 2016 a permis de faire une analyse de l'état de couverture végétale du sol. Sept unités d'occupation des terres ont été identifiées et représentées à savoir: les champs ou encore les zones de culture, les formations ripicoles, les plans d'eau, la savane arborée, la savane arbustive, la savane herbeuse et les sols nus. Au regard de ces unités d'occupation, force est de constater que 
les zones de cultures (champs) avec 52,31\% occupe majoritairement la zone de recherche. Ces surfaces, sous exploitation agricole, se sont vues défrichées de leur couvert végétal avec pour conséquence une importante régression de la végétation naturelle. Les activités anthropiques sont à l'origine de l'apparition et/ou l'augmentation de sol nu avec une superficie de $0,20 \%$ (Tableau 4).

Afin d'estimer les valeurs du facteur $C$ dans la zone de recherche, nous avons utilisé la régression entre deux valeurs extrêmes. Ces valeurs sont tirées du diagramme expérimental représenté dans la Figure 2 (Gitas, et al., 2009).

L'équation de la droite de régression trouvée est : $C=0.9167-\mathrm{NDVI}^{*} 1.1667$.

La carte du facteur de couverture végétale du sol $\mathrm{C}$ a été reclassée en cinq classes, allant des sols nus aux sols à couverture végétale dense (Carte 5).

Une superficie de 16 030,40 hectares correspondant à $8,27 \%$ de la superficie totale de la zone de recherche est couverte par des valeurs de couverture végétale comprise entre 0,69 à 1,1. Ces zones ont une couverture végétale très dense et sont moins vulnérables à l'érosion hydrique. Cependant, la classe à valeur de couverture végétale comprise entre 0,3 à 0,54 s'étend sur une superficie de 26 347,81 hectares soit $13,59 \%$ de la superficie totale de la zone a une couverture végétale très faible et est plus exposée à l'érosion. La zone de recherche est majoritairement représentée par une couverture végétale moyennement dense dont les valeurs varient entre 0,60 à 0,63 . Elle s'étend sur une superficie de 54032,38 hectares soit $27,87 \%$ de la superficie totale (Tableau 5).

\section{Le facteur de pratique antiérosive (P)}

La zone de recherche est à majorité aménagée avec une superficie de 102762,11 ha soit $52,31 \%$ de la superficie totale. Les zones aménagées sont représentées par l'ensemble des surfaces défrichées et mises en culture. Par opposé, les zones non aménagées sont représentées par la végétation naturelle où l'emprise humaine n'est pas encore manifeste.

Cette zone représente $47,69 \%$ de la superficie totale (Tableau 6).

Les résultats présentés détaillent d'une part le calcul et la spatialisation des différents facteurs de RUSLE (R, K, LS, C, P), qui d'autre part, seront utilisés comme données en entrée pour la modélisation de l'érosion hydrique des sols dans le secteur d'étude.

\section{La combinaison des facteurs}

La Figure 3 présente la carte du taux d'érosion hydrique obtenue par superposition des différentes cartes factorielles. Elle donne la synthèse des étapes de l'élaboration de la carte de l'érosion hydrique.

La Carte 7 est le résultat de la superposition des différents paramètres et représente la répartition du risque érosif dû aux seuls effets des facteurs naturels.

Elle n'indique pas seulement la perte du sol de chaque pixel, mais fournit aussi des informations sur les zones critiques vis-à-vis du risque de la perte en sol.

Cette carte fournit des informations synthétiques sur la nature, l'intensité et la répartition spatiale du phénomène, et permet donc d'identifier les zones les plus affectées par l'érosion hydrique.

L'érosion hydrique est relativement faible sur presque toute la zone de recherche (Tableau 7), avec une valeur moyenne de 0,26 t/ha/an. Les faibles valeurs vont de 0 à 1.57 $\mathrm{t} / \mathrm{ha} / \mathrm{an}$ et s'observent sur une superficie de 187196,54 ha soit $97,92 \%$ de la superficie totale. Cependant, il faut noter l'existence de taches d'érosion intense dont les valeurs varient entre 8,42 et $36,35 \mathrm{t} / \mathrm{ha} / \mathrm{an}$ qui occupent une superficie de 362,11 ha soit 0,19 $\%$ et se répartissent sur l'ensemble de la zone de recherche.

Le Tableau 8 montre le niveau de corrélation des différents facteurs. Elle est très forte entre le risque érosif et respectivement les facteurs LS, C et K. Elle est relativement moins forte entre le risque érosif et le facteur R. 


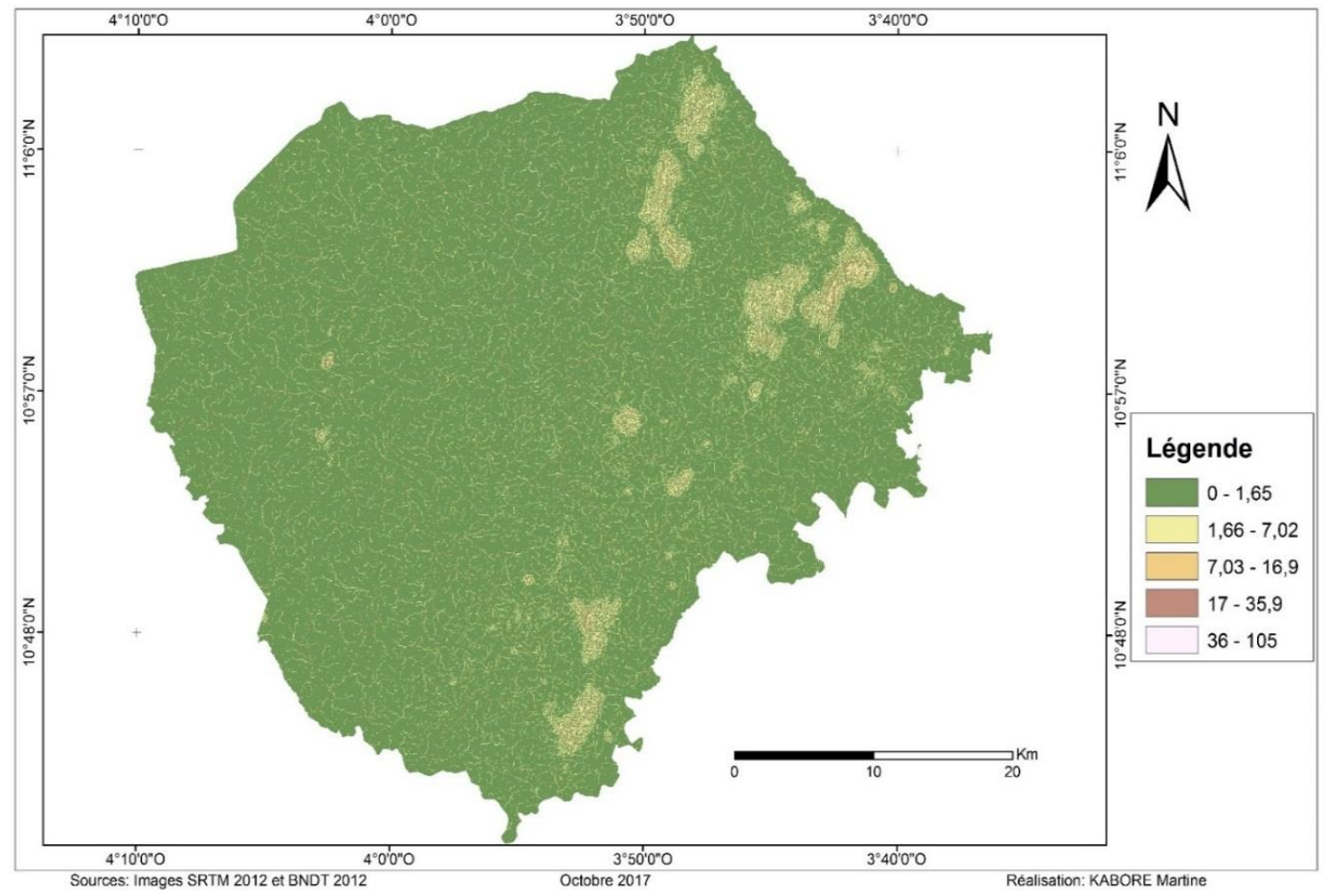

Carte 2 : Carte des longueurs de pente (LS).

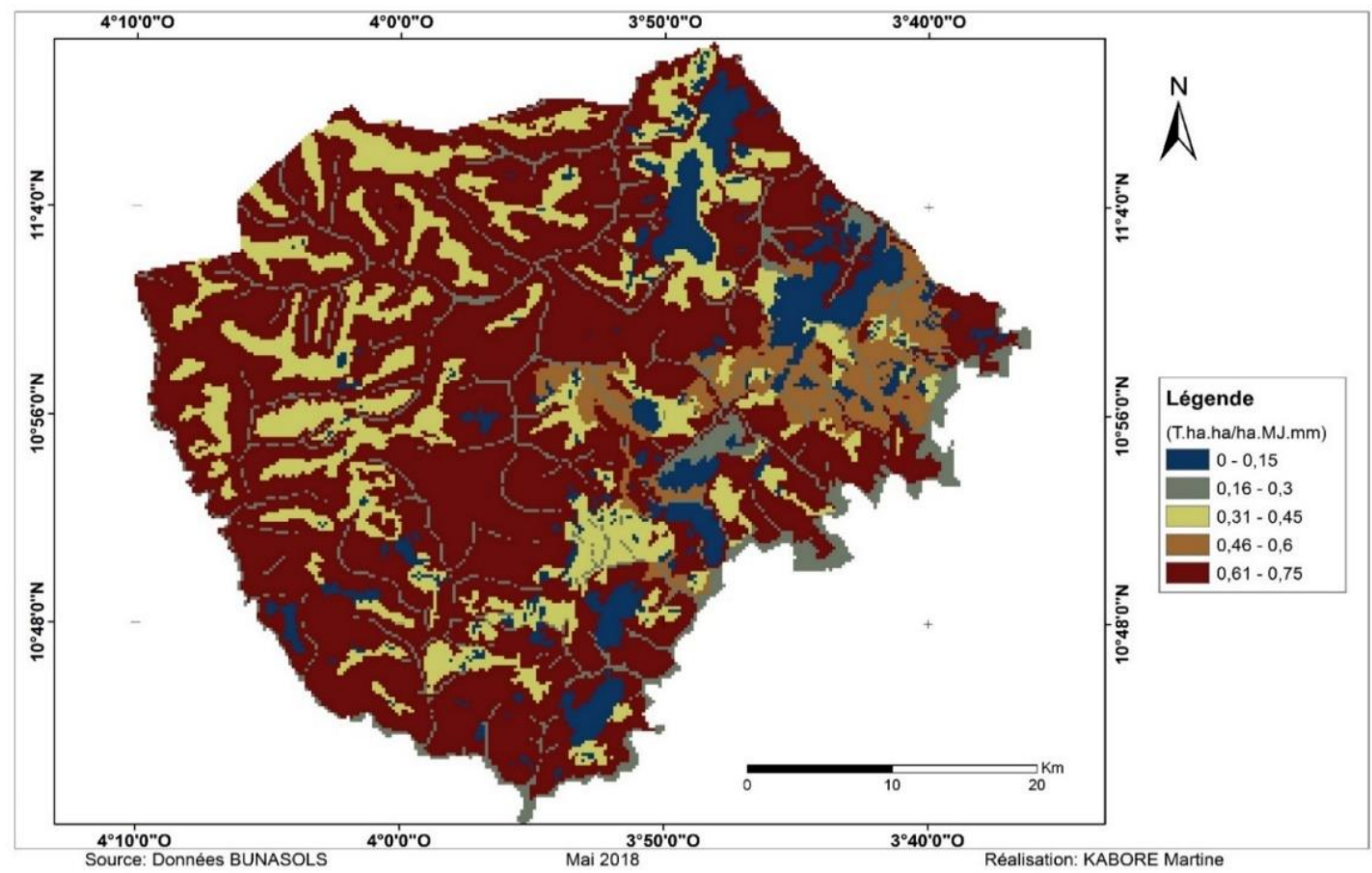

Carte 3 : Erodibilité (K) des sols. 
Tableau 2: Répartition des classes du facteur LS dans la zone de recherche.

\begin{tabular}{llll}
\hline $\begin{array}{l}\text { Classe du facteur } \\
\text { LS }\end{array}$ & Niveau d'appréciation & Superficie (ha) & Pourcentage (\%) \\
\hline $0-1,65$ & Très Faible & 179287,60 & 92,49 \\
$1,66-7,02$ & Faible & 11257,62 & 5,81 \\
$7,03-16,9$ & Moyen & 2506,03 & 1,29 \\
$17-35,9$ & Elevé & 670,37 & 0,35 \\
$36-105$ & Très élevé & 126,75 & 0,07 \\
& Total & 193848,37 & 100 \\
\hline
\end{tabular}

Source : Traitement d'image SRTM 2012

Tableau 3: Superficie des classes d'érodibilité K dans la commune de KarangassoVigué.

\begin{tabular}{llll}
\hline Classe d'érodibilité $\left(\mathbf{t} \mathbf{h ~ h a ~} \mathbf{~ M J}^{\mathbf{- 1}} \mathbf{h a}^{\mathbf{- 1}} \mathbf{m m}^{\mathbf{- 1}}\right)$ & Niveau d'appréciation & Superficie (ha) & pourcentage \\
\hline $0,61-0,75$ & Très élevé & 118231,94 & 60,64 \\
$0,46-0,6$ & Elevé & 10882,51 & 5,58 \\
$0,31-0,45$ & Moyen & 34113,77 & 17,50 \\
$0,16-0,3$ & Faible & 15021 & 7,70 \\
$0-0,15$ & Très faible & 16708,77 & 8,57 \\
& total & 194957,99 & 100 \\
\hline
\end{tabular}

Source : Données BUNASOLS, 2012

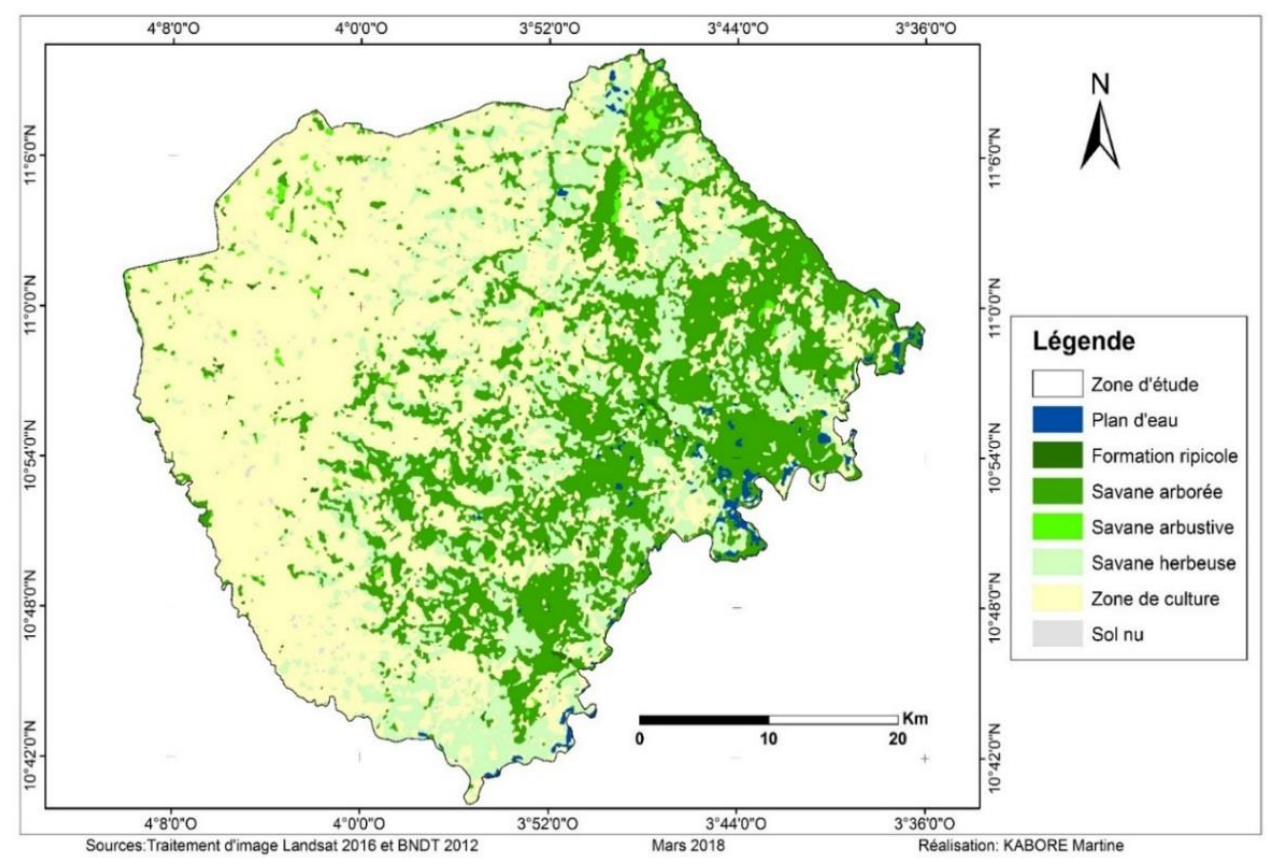

Carte 4 : Occupation des sols de la commune de Karangasso-Vigué en 2016. 
Tableau 4 : Occupation des terres en 2016.

\begin{tabular}{lll}
\hline Unité d'occupation & Superficie (ha) & Pourcentage (\%) \\
\hline Formation ripicole & 661,93 & 0,34 \\
Plan d'eau & 1934,82 & 0,98 \\
Savane arborée & 53220,43 & 27,09 \\
Savane arbustive & 2051,90 & 1,04 \\
Savane herbeuse & 35440,07 & 18,04 \\
Sol nu & 390,87 & 0,20 \\
Zone de culture & 102762,11 & 52,31 \\
Total & 196462,12 & 100,00 \\
\hline
\end{tabular}

Source: image Landsat, 2016.

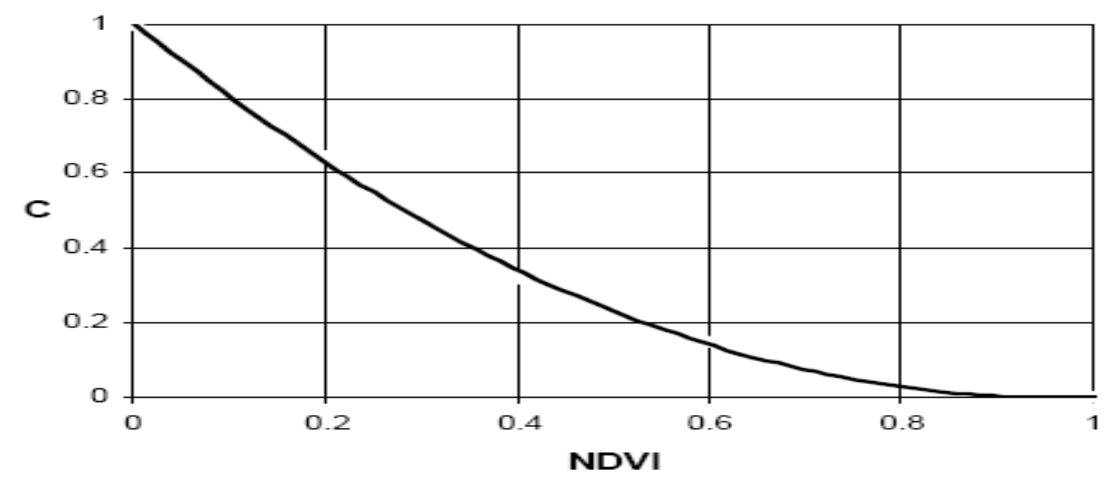

Figure 2: Diagramme expérimental d'estimation du facteur C (Gitas et al., 2009).

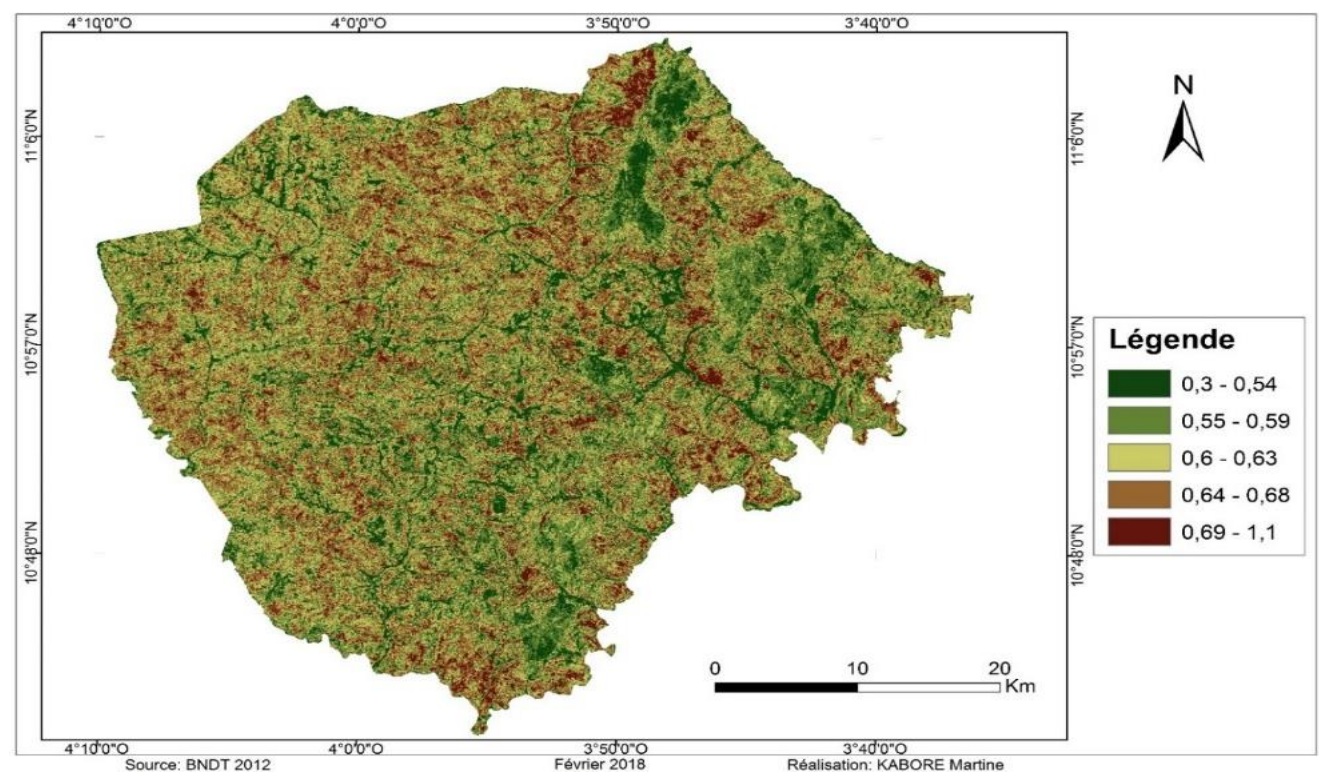

Carte 5 : Facteur de l'occupation des sols C. 
Tableau 5 : Superficie des différentes classes du facteur C.

\begin{tabular}{llll}
\hline Niveau d'appréciation & $\begin{array}{l}\text { Classe de valeur } \\
\text { du facteur C }\end{array}$ & Superficie (ha) & Pourcentage \\
\hline Très faible & $0,3-0,54$ & 26347,81 & 13,59 \\
Faible & $0,55-0,59$ & 49467,92 & 25,51 \\
Moyen & $0,6-0,63$ & 54032,38 & 27,87 \\
Dense & $0,64-0,68$ & 48007,13 & 24,76 \\
Très dense & $0,69-1,1$ & 16030,40 & 8,27 \\
Total & & 193885,64 & 100 \\
\hline Source : image Landsat, 2016. & & &
\end{tabular}

Tableau 6 : Superficie des différentes classes du facteur P.

\begin{tabular}{lll}
\hline Type de zone & Superficie (ha) & Pourcentage (\%) \\
\hline Zone aménagée & 102762,11 & 52,31 \\
Zone non aménagée & 93700,01 & 47,69 \\
Total & 196462,12 & 100 \\
\hline
\end{tabular}

Source : Image Landsat, 2016.

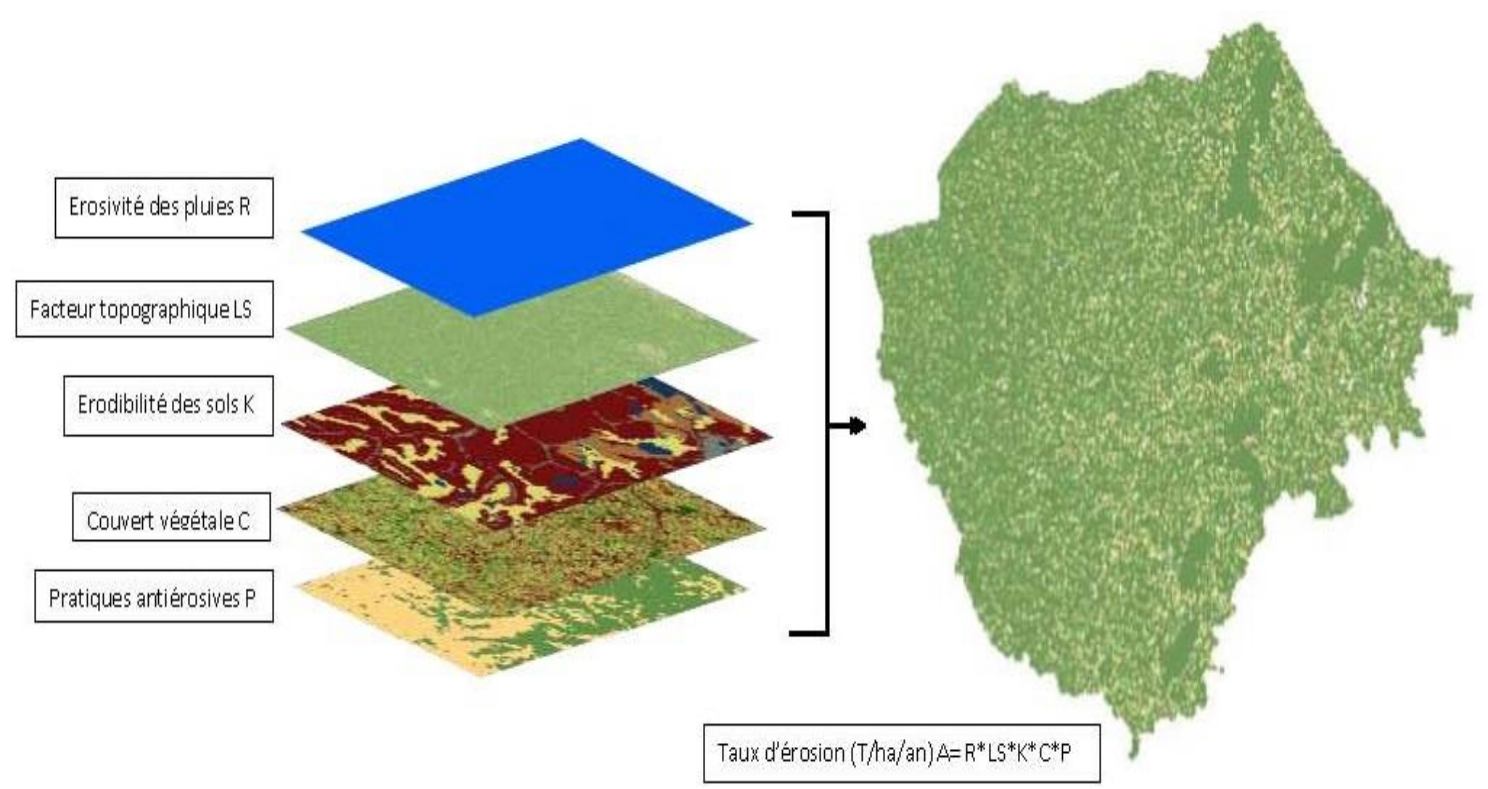

Figure 3 : Synthèse des étapes d'élaboration de la carte du taux d'érosion. 


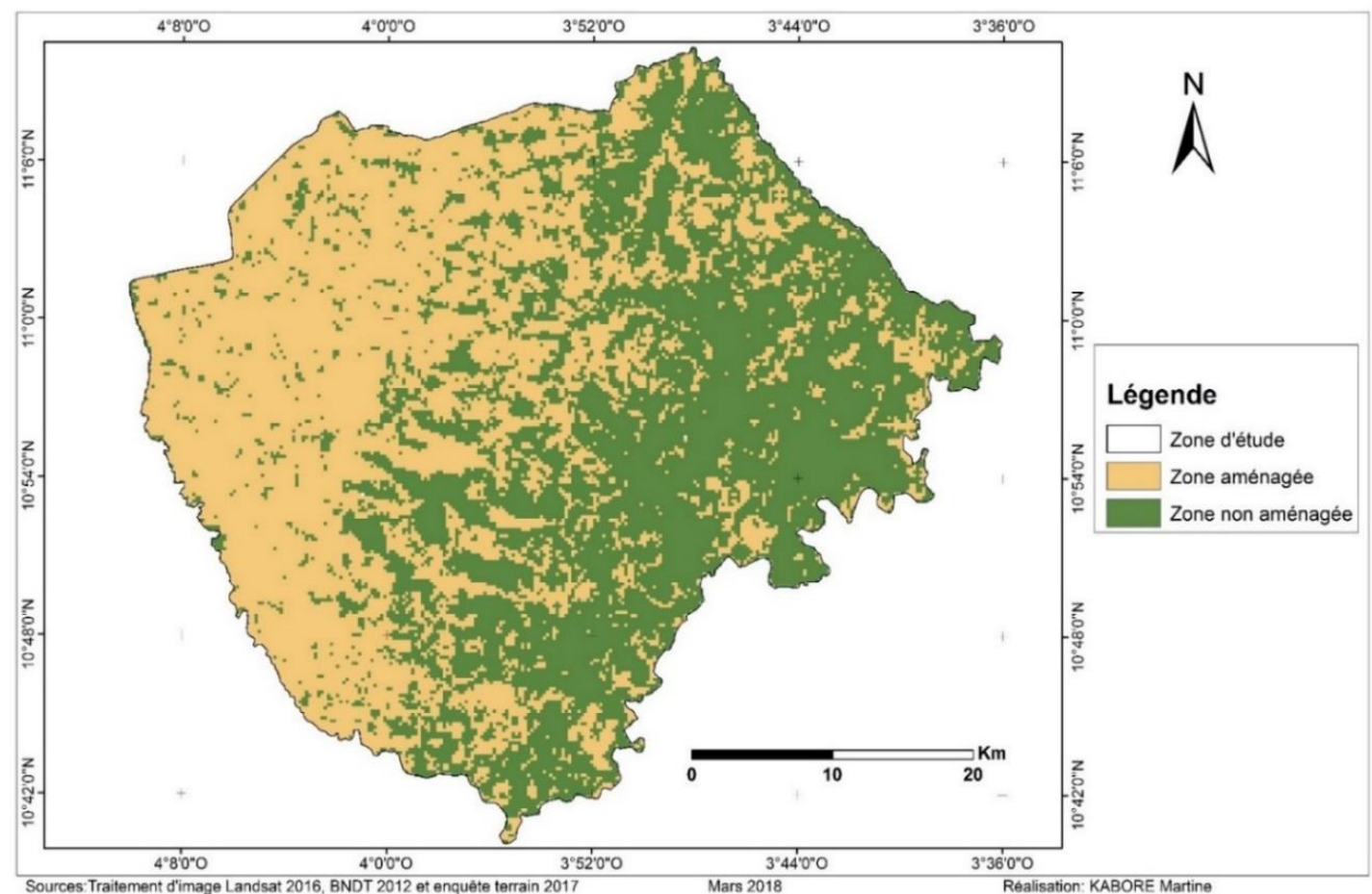

Carte 6: Facteur de pratique anti-érosive.

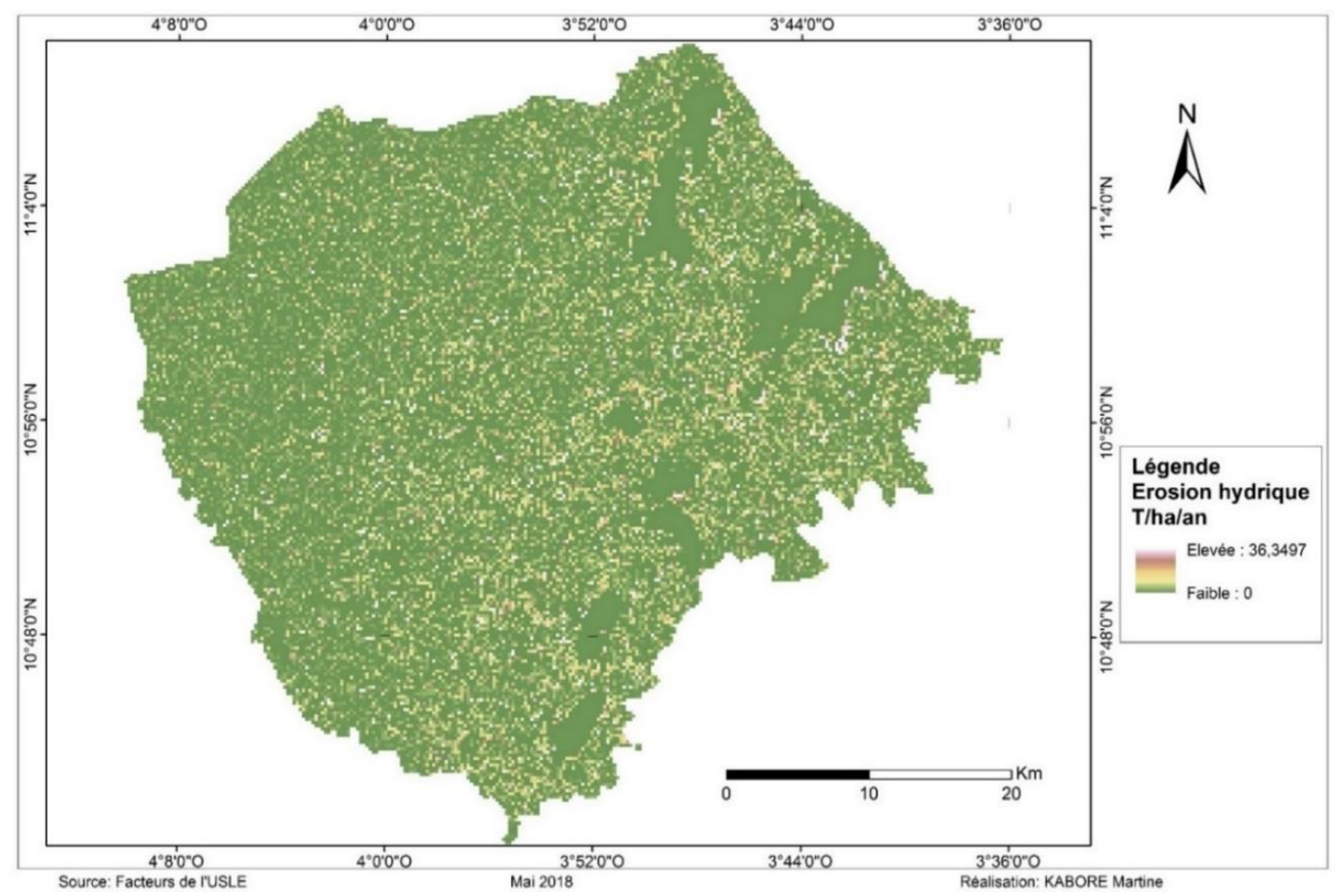

Carte 7 : Potentiel d'érosion hydrique. 
Tableau 7 : Quantification de l'érosion hydrique.

\begin{tabular}{llll}
\hline $\begin{array}{l}\text { Classe de risque } \\
\text { d'érosion du sol }\end{array}$ & $\begin{array}{l}\text { Perte en terre en } \\
\text { tonnes/hectare/an }\end{array}$ & Superficie (ha) & Pourcentage (\%) \\
\hline Faible & $0-1,57$ & 187196,54 & 97,92 \\
Modéré & $1,58-8,41$ & 3618,31 & 1,89 \\
Elevé & $8,42-36,35$ & 362,11 & 0,19 \\
& Total & 191176,97 & 100 \\
\hline
\end{tabular}

Source : résultats issus du modèle

Tableau 8: Corrélation spatiale entre les facteurs.

\begin{tabular}{llllllll}
\hline Variables & Risque érosif & Facteur R & Facteur K & Facteur C & $\begin{array}{l}\text { Facteur } \\
\text { LS }\end{array}$ & Pente & NDVI \\
\hline $\begin{array}{l}\text { Risque } \\
\text { érosif }\end{array}$ & 1 & & & & & & \\
Facteur R & 0,485 & 1 & & & & & \\
Facteur K & 0,844 & 0,879 & 1 & & & & \\
Facteur C & 0,845 & 0,878 & 1,000 & 1 & & & \\
Facteur LS & 1,000 & 0,488 & 0,845 & 0,847 & 1 & & \\
Pente & 1,000 & 0,502 & 0,854 & 0,855 & 1,000 & 1 & \\
NDVI & 0,881 & 0,841 & 0,997 & 0,997 & 0,883 & 0,890 & 1 \\
\hline
\end{tabular}

\section{DISCUSSION}

Le modèle de Wischmeier et Smith est basé sur des données de parcelles ou de bassin versant de très petite surface. La mise à l'échelle par le Système d'Information géographique pose la problématique de la précision des données à collecter. Quelques questions subsistent sur la méthode de collecte des données in situ et sur l'évaluation des méthodes de spatialisation pour assurer une bonne représentativité des paramètres et intrants du modèle. Pour chaque facteur d'érosion cartographié, la question de la précision reste toujours posée.

La cartographie du facteur d'érosivité s'est trouvée limitée par la faiblesse du maillage du réseau des postes pluviométriques. En effet, le secteur étudié ne dispose qu'une seule station, celle de Bobo Dioulasso, limitant ainsi la variabilité du facteur et de son influence sur les résultats. En s'appuyant sur les travaux de Roose et al. (2004), les pluies tropicales sont très agressives. Avec une seule station pluviométrique, l'érosivité des pluies serait uniforme et on considère dans ce cas que le facteur $\mathrm{R}$ est constant et égal à un (1) sur l'ensemble du site.

On pourrait penser à tester chacune des classes retenues par des expérimentations en se basant sur des observations in situ. Ces limites doivent être considérées et adaptées en intégrant les SIG qui permettent d'une part, de quantifier les pertes annuelles en sols et d'autre part, de cartographier les zones à vulnérabilité érosif.

Plusieurs auteurs ont abordé le phénomène de l'érosion hydrique des sols par analyse des images satellites, ils ont décelé différents facteurs de l'érosion hydrique et 
calculé la superficie de terre dénudée. Il s'agit des auteurs Sadiki et al. (2004), Payet et al. (2011), Ake et al. (2012), El Garouani et al. (2007), et Mesrar et al. (2015) qui en plus de l'utilisation des SIG pour le diagnostic des facteurs déterminants de l'érosion hydrique des sols, ont procédé à une modélisation du risque érosif. Les résultats obtenus dans cette étude sont en concordance avec les bases théoriques qui sous-tendent le choix des facteurs influençant les quantités de terres perdues par érosion hydrique. Comme l'ont montré, Warren et al. (2001), il s'agit, notamment des caractéristiques morpho pédologiques du milieu (faiblesse des pentes, tortuosité des axes d'écoulement, distribution des matériaux à l'intérieur du bassin). Les résultats montrent également qu'il est possible d'utiliser les données spatiales dans un cadre géo référencé suivant un modèle de référence comme l'équation universelle des pertes en terre (USLE) pour la zone soudanienne du Burkina Faso.

Malgré les critiques adressées sur l'utilisation abusive de l'USLE dans des conditions autres que celles où elle a été élaborée, il a été montré qu'en pratique, l'approche de modélisation par l'emploi des facteurs de RUSLE demeure une stratégie acceptable pour évaluer l'érosion hydrique des sols (Wall et al., 2002 ; El Hafid et al., 2012).

\section{Conclusion}

La présente étude met en évidence l'aspect quantitatif et qualitatif des pertes en terre, à travers le modèle RUSLE (équation Universelle Révisée de Perte en Sol) dont les paramètres ont été essentiellement estimés par l'utilisation des SIG et des images de la télédétection. La cartographie des zones d'érosion hydrique de la commune de Karangosso Vigué à travers l'équation RUSLE a permis de distinguer trois classes de vulnérabilité à l'érosion hydrique. Les zones à faible vulnérabilité qui couvrent $97 \%$ du secteur d'étude, les zones à moyenne vulnérabilité soit $0,26 \%$ et celles à forte vulnérabilité représentant $0,19 \%$. Les statistiques montrent que le territoire d'étude n'est pas très soumis à une érosion intensive.
Cependant, le rythme des défrichements et les systèmes miniers de production n'augurent pas de lendemain meilleur pour les agriculteurs. Cette étude constitue une contribution dans la connaissance des facteurs qui alimentent l'érosion dans la commune Karangassso Vigué à l'Ouest du Burkina Faso. Le recours à la géomatique est nécessaire pour l'évaluation des pertes en terre, car la spatialisation des paramètres permet d'élargir les zones d'intérêt qui peuvent dépasser le champ expérimental et considérer des régions ou bassins versants. La carte de vulnérabilité à l'érosion hydrique obtenue à l'issue de ce travail, pourrait constituer un premier document d'orientation en vue d'une utilisation rationnelle des sols de la région. $\mathrm{La}$ spatialisation du modèle de perte de terre sous environnement SIG et de télédétection a donné des résultats dont la validation reste nécessaire.

\section{CONFLIT D'INTERETS}

Les auteurs déclarent qu'ils n'ont aucun conflit d'intérêts pour cet article.

\section{CONTRIBUTIONS DES AUTEURS}

Les auteurs ci-dessous ont participé de diverses manières à la conception de cet article. La conception de la méthodologie et du choix des sites d'étude ont été effectués sur la supervision de $\mathrm{OB}$, investigateur principal. La collecte de données terrain a été effectuée par KM. Les analyses statistiques par KO et la correction du manuscrit ont été réalisées avec la collaboration de $\mathrm{KO}$ et de $\mathrm{KM}$.

\section{REMERCIEMENTS}

Les auteurs remercient tous les acteurs impliqués dans cette étude. Nos remerciements s'adressent particulièrement à la population de la commune de Karangasso Vigué et aux agents des services déconcentrés de l'Etat pour avoir prêté attention à notre questionnaire.

\section{REFERENCES}

Ake GE, Kouadio BH, Adja MG, Ettien JB, Effebi KR, Biémi J. 2012. Cartographie de la vulnérabilité multifactorielle à 
l'érosion hydrique des sols de la région de Bonoua (Sud-Est de la Côte d'Ivoire). Physio-Géo, 6: 23-42. DOI: 10.4000/physio-geo.2285

Avakoudjo J, Kouelo AF, Kindomihou V, Ambouta K, Sinsin B. 2015. Effet de l'érosion hydrique sur les caractéristiques physicochimiques du sol des zones d'érosion (dongas) dans la Commune de Karimama au Bénin. Agronomie Africaine, 27(2): 127-143.

Da DEC. Yacouba H Et Yonkeu S, 2008 : Unités morpho pédologiques et gestion de la fertilité des sols dans le CentreNord du Burkina Faso par les populations locales. Int. J. Biol. Chem. Sci., 2(3): 306-315. http://ajol.info/index.php/ijbcs

El Garouani A, Chen H, Lewis L, Tribak A, Abahrour M. 2007. Apport de la Télédétection et du SIG pour le suivi spatio-temporel de l'occupation du sol et de l'érosion nette dans le bassin de l'Oued Tlata (Maroc). Actes des JSIRAUF, Hanoi, p. 6-9.

Elhafid D, Akdim B. 2018. Quantification de l'érosion hydrique en utilisant le modèle RUSLE et déposition intégrée dans un SIG. Cas du bassin versant de l'oued isly (Maroc Oriental). European Scientific Journal, 14(5). DOI: 10.19044/esj.2018.v14n5p373

Fournier J, Serpantiè G, Delhoume JP, Gathelier R. 2000. Rôle des jachères sur les écoulements de surface et l'érosion en zone soudanienne du Burkina Faso: Application à la gestion des terres cultivées. Sud Sciences et Technologies, $\quad \mathbf{5}(1) . \quad$ DOI : http://dx.doi.org/10.4314/sset.v5i1.1830 1

Gasse F. 2006. Climate and Hydrological changes in tropical Africa during the past millions years. Comptes Rendus Palevol, 5(1-2): 35-43. DOI: 10.1016/j.crpv.2005.09.012

Gitas ZI, Douros K, Minakou C, Silleos GN, Karydas CG. 2009: Multi-temporal soil erosion risk assessment in $\mathrm{n}$. Chalkidiki using a modified usle raster model,
EARSeL eProceedings 8, 1/2009 p 4052.

Gonin A, Tallet B, 2012. Quel avenir pour l'élevage dans le bassin cotonnier de L'ouest du Burkina Faso? Dynamiques agro pastorales et recompositions territoriales. Presses de Sciences Po. DOI :

https://doi.org/10.3917/autr.060.0095

Hountondji HYC. 2008. Dynamique environnementale en zones sahélienne et soudanienne de l'Afrique de l'Ouest: analyse des modifications et évaluation de la dégradation du couvert végétal. Thèse de Doctorat, Université de Liège (Belgique), p. 153.

Hussein El Hage Hassan, Charbel L, Touchart L. 2015. Cartographie des conditions de l'érosion hydrique des sols au Mont-Liban : exemple de la région d'El Aaqoûra. Physio-Geo. URL: http://journals.openedition.org/physiogeo/4572; DOI: $10.4000 /$ physiogeo.4572.

Kohio EN, Toure AG, Sedogo MP, Ambouta KJM. 2017. Contraintes à l'adoption des bonnes pratiques de Gestion Durable des Terres dans les zones soudaniennes et soudano-sahéliennes du Burkina Faso. Int. J. Biol. Chem. Sci., 11(6): 2982-2989. DOI: https://dx.doi.org/10.4314/ijbcs.v11i6.3 4

Mesrar H, Sadiki A, Navas A, Faleh A, Quijano L, Jamal C. 2015 : Modélisation de l'érosion hydrique et des facteurs causaux, Cas de l'oued Sahla, Rif Central, Maroc. Annales de Geomorphologie, 59(4): 495 - 514. DOI: $10.1127 / \mathrm{zfg} / 2015 / 0169$.

Mokhtari E. 2017. Impact de l'érosion hydrique sur l'envasement du barrage Ghrib. Thèse de Doctorat troisième cycle d'hydrologie, Université Hassiba Benbouali de Chlef, p.272.

Nana PP. 2018. Du groupe à l'individu : dynamique de la gestion foncière en pays gouin (sud-ouest du Burkina Faso). Belgeo URL http://journals.openedition.org/belgeo/2 
6080 ; DOI : 10.4000/belgeo.26080

Ouandaogo N, Ouattara B, Pouya MB, Gnankambary Z, Nacro HB, Sedogo PM. 2016. Effets des fumures organominérales et des rotations culturales sur la qualité des sols. Int. J. Biol. Chem. Sci., 10(2): 904-918. DOI http://dx.doi.org/10.4314/ijbcs.v10i2.37

Ouédraogo B, Ouédraogo L, Kaboré O. 2015. Fragmentation de l'espace et conflits d'usage au sahel : cas du bassin versant de Yakouta (Burkina Faso). Int. J. Biol. Chem. Sci., 9(6): 2727-2739. DOI: http://dx.doi.org/10.4314/ijbcs.v9i6.17

Ozer P, Hountondji Y, Niang AJ, Karimoune S, Laminou Manzo O, Salmon M. 2010. Désertification au Sahel: historique et perspectives. Bulletin de la Société Géographique de Liège, 54 : 69-84.

Payet E, Dumant P, Pennober G. 2011. Modélisation de l'érosion hydrique des sols sur un bassin versant du Sud-Ouest de Madagascar. Le Fiherenana, 11(3) : 26p.

URL: http://journals.openedition.org/vertigo/1 2591; DOI: 10.4000/vertigo.12591

Roose E, De Noni G. 2004. Recherches sur l'érosion hydrique en Afrique: revue et perspectives. Sécheresse, 15(1): 121129.

Sadiki A, Bouhlassa S, Auajjar J, Faleh A, Macaire JJ. 2004. Utilisation d'un SIG pour l'évaluation et la cartographie des risques d'érosion par l'Équation universelle des pertes en sol dans le Rif oriental (Maroc) : cas du bassin versant de l'oued Boussoua. Bulletin de l'Institut Scientifique, Rabat, section Sciences de la Terre, 26: 69-79.
Simonneaux V, Cheggour A, Deschamps C, Mouillot F, Cerdan, Le Bissonnais Y. 2015. Land use and climate change effects on soil erosion in a semi-arid mountainous watershed (High Atlas, Morocco). Journal of Arid Environments, 122: 64-75. DOI : https://doi.org/10.1016/j.jaridenv.2015. 06.002 .

Wall GJ, Coote DR, Pringle EA, Shelton IJ. 2002. RUSLE-CAN Équation universelle révisée des pertes de sol pour application au Canada. Manuel pour l'évaluation des pertes de sol causées par l'érosion hydrique au Canada. Direction générale de la recherche, Agriculture et Agroalimentaire Canada, $\mathrm{N}^{\circ}$ de la contribution AAC2244F, $117 \mathrm{p}$.

Warren A, Batterbury S, Osbahr H. 2001. Soil erosion in the West African Sahel: a review and an application of a local political ecology approach in south west Niger. Global Environmental Change, 11: 79-95. https://doi.org/10.1016/S09593780(00)00047-9

Wischmeier WH, Smith DD. 1978. Predicting rainfall erosion losses. A guide to conservation planning. Agriculture Handbook (vol. 537). Édit. US Department of Agriculture; p.58.

Yjjou M, Bouabid R, El Hmaidi A, Essahlaoui A. 2014. Modélisation de l'érosion hydrique via les SIG et l'équation universelle des pertes en sol au niveau du bassin versant de l'Oum Er-Rbia. The International Journal of Engineering and Science (IJES), 3(8): 83-91. 